\title{
The bacterial enhancer-binding protein NTRC is a molecular machine: ATP hydrolysis is coupled to transcriptional activation
}

\author{
Andrew Wedel $^{1}$ and Sydney Kustu \\ Departments of Plant Biology and Molecular and Cell Biology, University of California Berkeley, Berkeley, \\ California 94720 USA
}

NTRC is a prokaryotic enhancer-binding protein that activates transcription by $\sigma^{54}$-holoenzyme. NTRC has an ATPase activity that is required for transcriptional activation, specifically for isomerization of closed complexes between $\sigma^{54}$-holoenzyme and a promoter to open complexes. In the absence of ATP hydrolysis, there is known to be a kinetic barrier to open complex formation (i.e., the reaction proceeds so slowly that the polymerase synthesizes essentially no transcripts even from a supercoiled template). We show here that open complex formation is also thermodynamically unfavorable. In the absence of ATP hydrolysis the position of equilibrium between closed and open complexes favors the closed ones. Use of linear templates with a region of heteroduplex around the transcriptional start site-_"preopened" templates-does not bypass the requirement for either NTRC or ATP hydrolysis, providing evidence that the rate-limiting step in open complex formation does not lie in DNA strand denaturation per se. These results are in contrast to recent findings regarding the ATP requirement for initiation of transcription by eukaryotic RNA polymerase II; in the latter case, the ATP requirement is circumvented by use of a supercoiled plasmid template or a preopened linear template.

[Key Words: $\sigma^{54}$; open complexes; heteroduplex template; enteric bacteria; enhancers; ATPase]

Received January 13, 1995; revised version accepted June 28, 1995

NTRC (nitrogen regulatory protein C) is an enhancerbinding protein that activates transcription by an alternative holoenzyme form of RNA polymerase containing $\sigma^{54}$ as the $\sigma$ factor (Fig. 1 ; for review, see Kustu et al. 1991; Magasanik 1993; Porter et al. 1995). To activate transcription NTRC must hydrolyze ATP or GTP. The requirement for an activator capable of hydrolyzing a nucleoside triphosphate appears to be universal for transcription by $\sigma^{54}$-holoenzyme (for review, see Kustu et al. 1989; Thony and Hennecke 1989|. The activators, all of which bind to sites distant from promoters, allow transcription in response to different physiological signals. A well-studied promoter recognized by $\sigma^{54}$-holoenzyme is that for the $g \ln A$ gene of enteric bacteria, which encodes glutamine synthetase. Transcription from the $g \ln A$ promoter is activated by NTRC in response to limitation of combined nitrogen.

$\sigma^{54}$-Holoenzyme can bind to the $g \ln A$ promoter in a closed recognition complex in the absence of other factors but requires the presence of NTRC and ATP to isomerize into the open complex, in which the DNA around the transcriptional start site is denatured locally

${ }^{1}$ Present address: Max-Planck-Institut fuer Biochemie, 82152 Martinsreid, Germany.
(Fig. 1; Kustu et al. 1991). Neither ATP $\gamma$ S nor ADP can substitute for ATP in allowing formation of open complexes although both inhibit the ATPase activity of NTRC, indicating that they bind to the protein (Weiss et al. 1991). NTRC binds to an enhancer consisting of two tandem DNA-binding sites centered at -140 and -108 with respect to the transcriptional start site and contacts the polymerase by means of a DNA loop. To hydrolyze ATP and activate transcription, NTRC must be phosphorylated on an aspartic acid residue within its aminoterminal regulatory ("receiver") domain (Weiss and Magasanik 1988; Sanders et al. 1992). This phosphorylation, which is mediated by the NTRB protein, occurs under nitrogen-limiting conditions and is labile; the half-life of phosphorylated NTRC is $\sim 4 \mathrm{~min}$ at $37^{\circ} \mathrm{C}$.

A number of mutant forms of NTRC-so-called NTRC ${ }^{\text {constitutive }}$ proteins-have some ability to activate transcription without being phosphorylated (Popham et al. 1989; Weglenski et al. 1989; Dixon et al. 1991; Klose et al. 1993; Flashner et al. 1995). All of these forms also have the ability to hydrolyze ATP without being phosphorylated and depend on nucleotide hydrolysis to catalyze the formation of open complexes by $\sigma^{54}$-holoenzyme in vitro. The latter findings distinguish the nucleotide requirement for open complex formation from that 

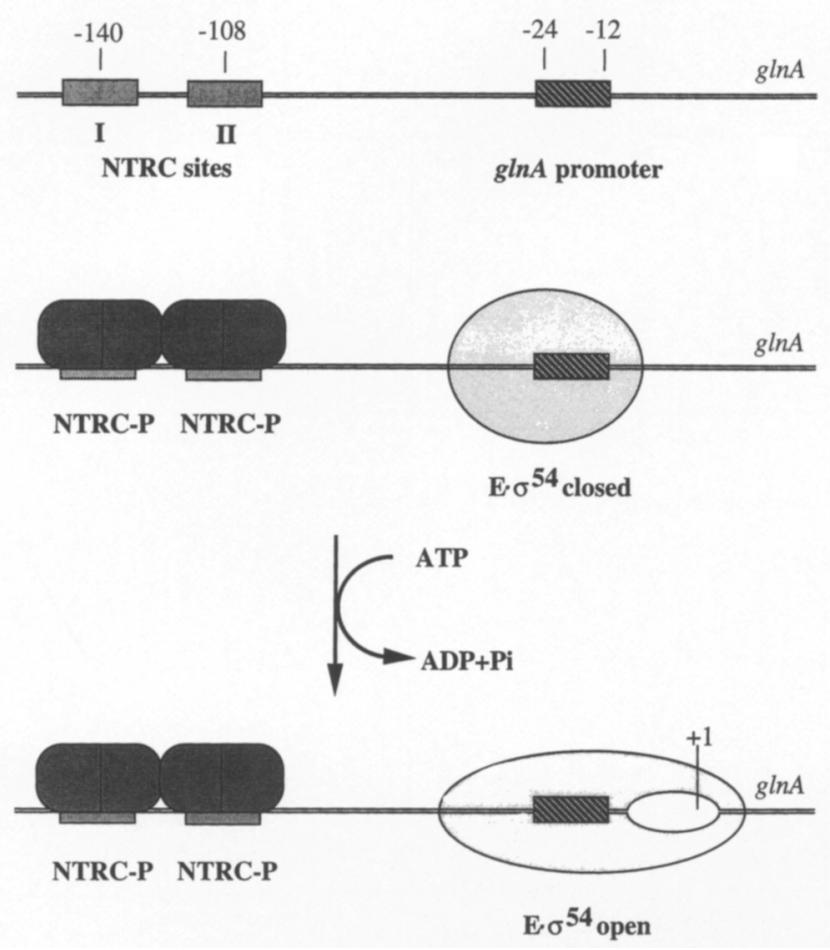

Figure 1. Formation of open complexes at the $g \ln A$ promoter (for review, see Kustu et al. 1991; Porter et al. 1995). o $^{54}$-Holoenzyme can bind to the $g \ln A$ promoter in a closed recognition complex in the absence of other factors. Conserved promoter sequences lie in the -24 and -12 regions with respect to the start site of transcription. Dimers of NTRC bind to the enhancer, which is composed of two dyad-symmetrical sites centered at -140 and -108 . NTRC contacts the polymerase by means of a DNA loop (not shown). When phosphorylated, NTRC catalyzes the isomerization of a closed complex between $\sigma^{54}$-holoenzyme and the promoter to a transcriptionally competent open complex, in which the DNA around the transcriptional start site is denatured. To catalyze open complex formation, NTRC must hydrolyze ATP. In open complexes, the conformation of polymerase is changed such that its DNase I footprint is elongated and extends downstream of the transcriptional start site (Popham et al. 1989).

for phosphorylation. Unlike phosphorylated wild-type NTRC, NTRC ${ }^{\text {constitutive }}$ proteins provide a homogenous population of active molecules.

There are two striking features that distinguish $\sigma^{54}$ from other $\sigma$ factors. First, $\sigma^{54}$ bears no detectable sequence homology to other $\sigma$ factors (Merrick 1993), all of which are homologous to one another (Hellman and Chamberlin 1988; Lonetto et al. 1992). Second, conserved dinucleotides in $\sigma^{54}$-dependent promoters must be exactly one turn of the DNA helix apart $(10 \mathrm{bp})$, whereas conserved promoter elements for other holoenzyme forms of polymerase are 2 to $2 \frac{1}{2}$ turns apart. Moreover, $\sigma^{54}$ alone can bind to "strong" (high-affinity) promoters in the absence of core polymerase (Buck and Cannon 1992; Cannon et al. 1994).

In this paper we show that the barrier to open complex formation by $\sigma^{54}$-holoenzyme is thermodynamic, as well as kinetic. Because NTRC acts catalytically in the for- mation of open complexes, this indicates that the energy of ATP hydrolysis must be coupled to open complex formation to drive the reaction and hence that NTRC is a simple molecular machine (Alberts and Miake-Lye 1992). We also provide evidence that the rate-limiting step in open complex formation is not DNA strand denaturation per se.

\section{Results}

The barrier to open complex formation is both kinetic and thermodynamic

When an excess of $\sigma^{54}$-holoenzyme, a supercoiled template bearing the $g \ln A$ promoter, and an NTRC ${ }^{\text {constitutive }}$ protein (NTRC ${ }^{\mathrm{D} 5 \mathrm{E}}$, S160F) were incubated together in the absence of ATP, no detectable open complexes accumulated even after $60 \mathrm{~min}$ (Fig. 2A, lanes 1,2; assessed in a single-cycle transcription assay, see Materials and methods). However, when ATP was added at $60 \mathrm{~min}$, half of the promoters carried an open complex within $2 \mathrm{~min}$ (Fig. 2A, lane 3 and legend). If NTRC was subsequently prevented from hydrolyzing ATP by addition of the inhibitor ADP at $62 \mathrm{~min}$ (Weiss et al. 1991), the preformed open complexes slowly decayed (Fig. 2A, lanes 3-5, Fig. $2 \mathrm{~B} ; t_{1 / 2}$ for decay of $\sim 105 \mathrm{~min}$ ). Because no detectable open complexes formed spontaneously in the absence of ATP hydrolysis by NTRC, and the decay rate of open complexes on the $g \ln A$ promoter is very slow, there must be a kinetic barrier between the two forms. Therefore, the failure to detect formation of open complexes in the absence of ATP hydrolysis cannot be attributable to their lack of stability but must be attributable to their failure to form at all. Experiments in which open complexes were separated from ATP by sieving or were diluted to decrease the concentration of this nucleotide far below that required for open complex formation indicated that a slow rate of decay of open complexes did not depend on the presence of ATP or ADP and that the rate of decay was not affected by these nucleotides (Popham et al. 1989; Wedel 1993).

Even when an excess of $\sigma^{54}$-holoenzyme was incubated with the template in the presence of nucleotides to allow immediate synthesis of transcripts, essentially no transcripts were seen (Fig. 2A, lanes 6,7; Popham et al. 1989 ), indicating that open complexes do not form even transiently in the absence of NTRC. Any open complexes that had formed should have yielded transcripts because open complexes that are isolated away from NTRC subsequently are known to be transcriptionally competent (Popham et al. 1989; Popham 1989). Additional experiments indicated that $\sigma^{54}$-holoenzyme could not transcribe even in the presence of NTRC and all four nucleotides if the activator was prevented from hydrolyzing the $\beta-\gamma$ bond of purine nucleotides (Popham et al. 1989; Popham 1989; Wedel 1993). Hydrolysis of the $\beta-\gamma$ bond was blocked by the inhibitors ADP or ATP $\gamma S$ or, alternatively, by providing $\beta, \gamma$-methylene-ATP instead of ATP and using a low concentration of GTP.

As noted above (Fig. 2A, lanes 3-5; Fig. 2B), after inhibition of the ATPase activity of NTRC, the number of 
A

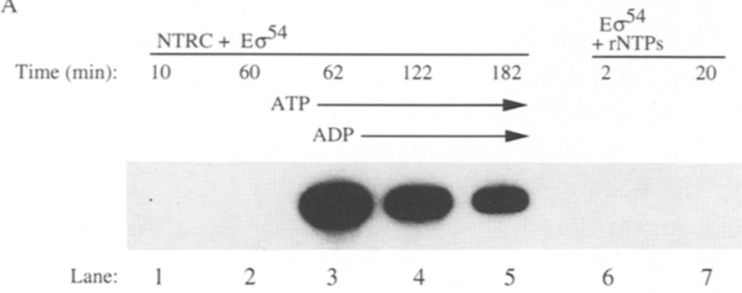

B

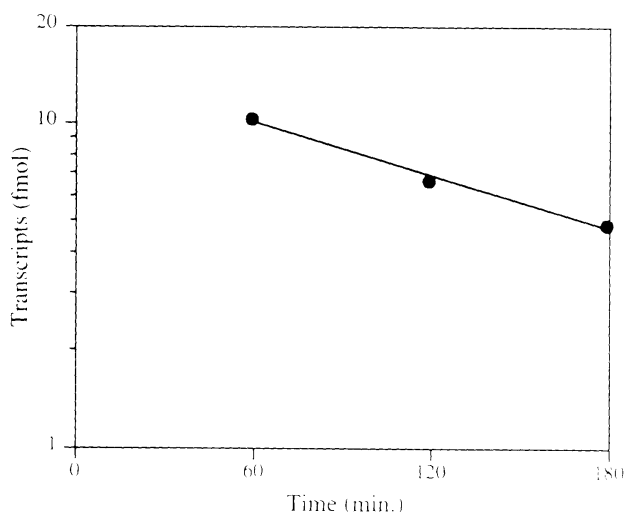

C Open complex formation

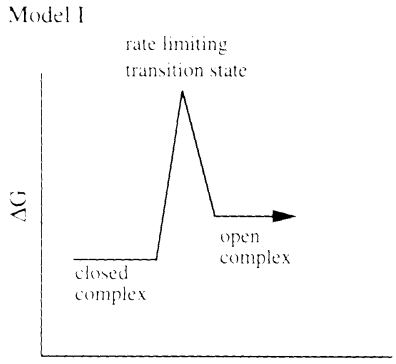

reaction coordinate

D Open complex decay

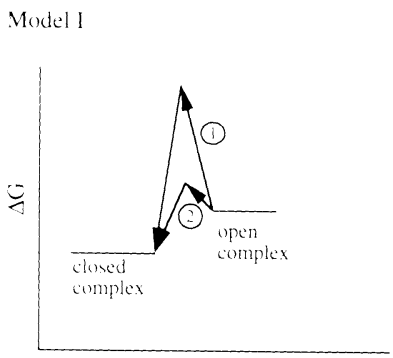

reaction coordinate

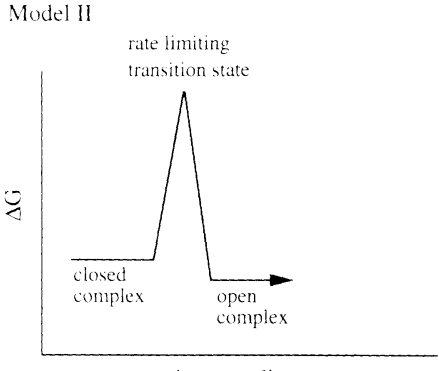

reaction coordinate

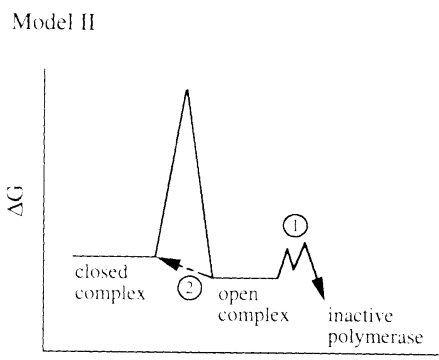

reaction coordinate

Figure 2. Requirements for the formation of open complexes $(A)$ and the time course of their decay $(B)$ indicate a kinetic barrier to open complex formation $\langle C, D|$. $|A\rangle)($ Lanes $1-5) \sigma^{54}$-Holoenzyme $\left(100 \mathrm{~nm}\right.$ core polymerase, $\left.400 \mathrm{nM} \sigma^{54}\right)$ and the NTRC $C^{\text {constitutive }}$ protein $\mathrm{NTRC}^{\mathrm{D} 5 \mathrm{E}}$, S160F $(200 \mathrm{nM}$; Klose et al. 1993) were incubated with a supercoiled template (pIES $463 ; 1 \mathrm{~nm}=20 \mathrm{fmoles} / 20 \mu l)$ bearing the wild-type $g \ln A$ promoter-regulatory region at $37^{\circ} \mathrm{C}$ in a final volume of $120 \mu \mathrm{l}$. At 10 and $60 \mathrm{~min} 20-\mu \mathrm{l}$ samples were taken (lanes 1,2$)$ to which ADP and heparin were added to $4 \mathrm{~mm}$ and $100 \mu \mathrm{g} / \mathrm{ml}$, respectively, to stop the subsequent formation of open complexes (see Results; Weiss et al. 1991); after 2 min at $37^{\circ} \mathrm{C}$, an elongation mix was added containing ATP, GTP, CTP, and $\left|\alpha^{-{ }^{32}} \mathrm{P}\right| \mathrm{CTP}$ to allow any polymerase molecules in open complexes to synthesize single transcripts. Immediately after the second sample was taken at 60 min, ATP was added to the remainder of the reaction mixture to $0.4 \mathrm{~mm}$. After incubation for $2 \mathrm{~min}$ at $37^{\circ} \mathrm{C}$, formation of open complexes was stopped by addition of ADP to $4 \mathrm{~mm}$ and a third sample of $20 \mu \mathrm{l}$ was taken to which the elongation mix was added (lane 3) (because the reaction mixture already contained ADP, only heparin was added before ribonucleotides). Under similar conditions, the formation of open complexes has been shown to cease within 2 min after the addition of ATP (Popham 1989). After 62 and 122 min further incubation in the presence of ATP and ADP (i.e., after 122 and $182 \mathrm{~min}$ total from the beginning of the experiment), two additional samples were taken to monitor the slow decay of open complexes (lanes 4,5). (Lanes 6,7$) \sigma^{54}$-Holoenzyme ( $100 \mathrm{nM}$ core polymerase and $400 \mathrm{nM}^{54}$ ) alone was incubated with the template in the presence of elongation mix and samples were taken for electrophoresis at 2 and $20 \mathrm{~min}$. Transcripts were isolated on a polyacrylamide gel as described in Materials and methods and the figure is an autoradiograph of the gel. $(B)$ Decay of open complexes. Transcripts in lanes 3-5 (10.2-, 6.6-, and 4.8-fmole transcripts, respectively, of a possible total of $20 \mathrm{fmole}$ if every template molecule had been used) were quantitated as described in Materials and methods and plotted on a logarithmic scale as a function of time. This yielded a half-life for open complexes of $\sim 105$ min. (C) Possible pathways for the formation of open complexes. Models I and II indicate that the overall change in free energy $(\Delta \Delta G)$ for the formation of open complexes can be positive or negative, respectively. $|D|$ Possible pathways for the decay of open complexes. For model I, the reverse of the forward pathway (pathway 1) and any number of other pathways (e.g., pathway 2) are consistent with the observations that (1) $\sigma^{54}$-holoenzyme does not form open complexes spontaneously, and (2) once open complexes are formed, they decay spontaneously. Open complexes can also decay to inactive polymerase (not indicated), but this is not informative. For model II, decay of open complexes to inactive polymerase (pathway 1) is also consistent with the above observations, whereas pathway 2 (indicated by a broken line) or any other pathway to closed complexes or active polymerase is not (see text).

open complexes between $\sigma^{54}$-holoenzyme and the $g \ln A$ promoter decreased with time, although there was a large excess of free holoenzyme present in solution. There are two classes of models to account for this decay (Figs. 2C,D). In the first class (e.g., model I), the barrier to open complex formation is thermodynamic as well as kinetic, that is, the standard free energy of the open complex is higher than that of the closed complex and hence the position of equilibrium favors closed complexes. In this case it should be possible to demonstrate that open complexes decay to the reactant, i.e., active $\sigma^{54}$-holoenzyme in closed complexes or in solution). This conclusion does not depend on the pathway for decay, which could be the reverse of the forward pathway or a different pathway (Fig. 2D). In the second class of model (e.g., model II), the barrier to open complex formation is only kinetic. Because the position of equilibrium favors open complexes, decay must be to inactive polymerase. The converse assumption, that decay could yield active polymerase in this case also, violates principles of thermo- 
dynamic reversibility. Any pathway for decay to active polymerase would require a net free energy change that was positive (Fig. 2D, broken line); hence, open complexes would form spontaneously by the reverse of this pathway more rapidly than they would decay. In both classes of models, open complexes do not form in the absence of ATP hydrolysis because the kinetic barrier between closed and open complexes is too high, regardless of the position of equilibrium.

Because closed complexes/active $\sigma^{54}$-holoenzyme have a measurable activity - that is, the ability to form open complexes-the models described above can be differentiated by asking whether the product formed when open complexes decay can be made to reform open complexes. To determine this, we first formed open complexes and separated them from free $\sigma^{54}$-holoenzyme by passage through a sieving column (diagram in Fig. 3A). The isolated open complexes were then allowed to decay, and the $\sigma^{54}$-holoenzyme released from them was given an opportunity to reform open complexes either on the same template or on a second template that yielded a transcript of different length. As detailed below, open complexes reformed in both cases (Fig. 3A, lanes 5-8, 9-12; quantified in Fig. 3B,Cl, indicating that decay was at least in part to active $\sigma^{54}$-holoenzyme.

The template used for the initial formation of open complexes was a linear fragment carrying a variant of the $\operatorname{gln} A$ promoter with four $\mathrm{A} / \mathrm{T}$ to $\mathrm{G} / \mathrm{C}$ transitions in the melting region; the half-life of open complexes on this template is $5-10 \mathrm{~min}$ at $37^{\circ} \mathrm{C}$ and $\sim 30 \mathrm{~min}$ at $4^{\circ} \mathrm{C}$. (We sought a short half-life at $37^{\circ} \mathrm{C}$ to minimize polymerase inactivation during the course of the experiment.) After rapid purification away from free $\sigma^{54}$-holoenzyme over a sieving column at $4^{\circ} \mathrm{C}$, open complexes were split into three portions. ATP was added back to all three, whereas NTRC and NTRB were added back to portions 2 and 3 only (diagram in Fig. 3A). To aliquot 3 was also added a 17-fold excess of a second template that yields a shorter transcript. The three samples were then placed at $37^{\circ} \mathrm{C}$ to allow simultaneous decay and reformation of open complexes. In the absence of NTRC, open complexes decayed from the first template with a half-life of $\sim 5$ min (Fig. 3A, lanes 1-4; Fig. 3B, open squares). However, in the presence of NTRC, many complexes reformed on the same template (Fig. 3A, lanes 5-8; Fig. 3B, solid squares). In the presence of NTRC and an excess of a second template, complexes decayed from the first template, initially with a 5-min half-life, and simultaneously reformed on the second (Fig. 3A, lanes 9-12; Fig. 3C, open and solid circles, respectively). The total number of open complexes on the two templates remained approximately constant over the course of the experiment (Fig. $3 \mathrm{C}$, open triangles), congruent with the view that open complexes formed on the second template derived from previous open complexes that had decayed from the first. Had there been free polymerase in the template fraction after sieving, both portions 2 and 3 would have had more open complexes than portion 1 at the zero time point. However, extrapolation of the two lines in Figure 3B to the zero time point shows that portion 2 , to which
NTRC was added, had no more open complexes than portion 1. Similarly, extrapolation of the line for the "Sum" in C to the zero time point and comparison with the extrapolation of either line in B indicates that there were no excess open complexes in portion 3 , in spite of the fact that it contained an additional template with a very high apparent affinity for polymerase (see Materials and methods and below; Wedel 1993). Results similar to those in Figure 3 were obtained in a second experiment in which template and/or NTRC and NTRB were added back after allowing different times for decay of open complexes from the first template (not shown). Because decay of open complexes was to reactant-active closed complexes or free $\sigma^{54}$-holoenzyme-the barrier to open complex formation is thermodynamic as well as kinetic (Fig. 2C, model I).

\section{Melting of the DNA around the transcriptional start site does not circumvent the requirement for either NTRC or ATP hydrolysis}

Because the barrier to open complex formation lies between closed complexes and localized DNA strand denaturation around the transcriptional start site, it is possible that $\sigma^{54}$-holoenzyme alone cannot carry out the melting reaction, and that NTRC catalyzes open complex formation by acting as a site-specific helicase. To ask whether strand denaturation was, in fact, the ratelimiting step in open complex formation, we constructed and tested several templates carrying $g \ln A$ promoter sequences in which the DNA duplex around the transcriptional start site was destabilized by base mispairing (i.e., "preopened" templates) (Fig. 4A). The region that is denatured in natural open complexes at $\sigma^{54}$-dependent promoters extends from approximately position -7 to position +3 or +4 with respect to the start site of transcription (Sasse-Dwight and Gralla 1988; Whitehall et al. 1992). The three templates that we tested had regions of heteroduplex from -10 to $-2,-1$ to +4 , and -10 to +4 , respectively; note that the denatured region in the third heteroduplex is longer than that in natural open complexes.

In the presence of NTRC and ATP, all of the promoters carrying regions of heteroduplex were used with comparable efficiency to one another and to the wild-type $g \ln A$ promoter (Fig. 4B, cf. lanes 3, 5, and 7 with lane 1), whereas in the absence of NTRC they were used poorly (lanes 4,6 , and 8). However, in contrast to the $g \ln A$ promoter, each of the promoters carrying a region of heteroduplex did support a small amount of transcription in the absence of NTRC. For the promoters carrying heteroduplex from -1 to +4 and -10 to +4 , transcription in the absence of NTRC was $1 \%-2 \%$ of that obtained with NTRC (not visible in Fig. 4B), whereas for the promoter carrying heteroduplex from -10 to -2 , this value was $5 \%-10 \%$. We have obtained three lines of evidence that transcription in the absence of NTRC results from the fact that $\sigma^{54}$-holoenzyme can initiate transcription without going through the normal open complex intermediate when presented with the unusual DNA struc- 
Wedel and Kustu

A

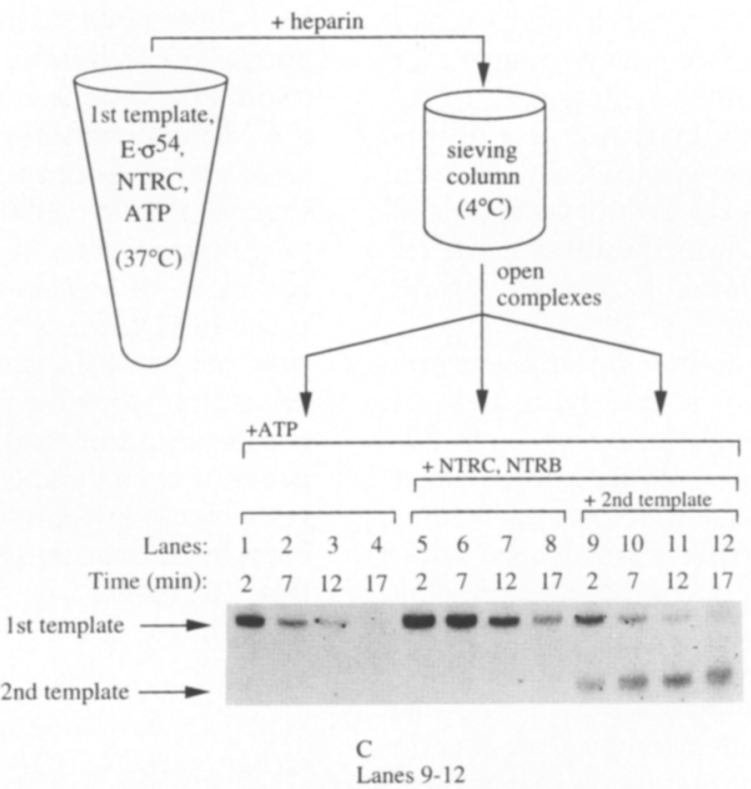
Lanes 1-8

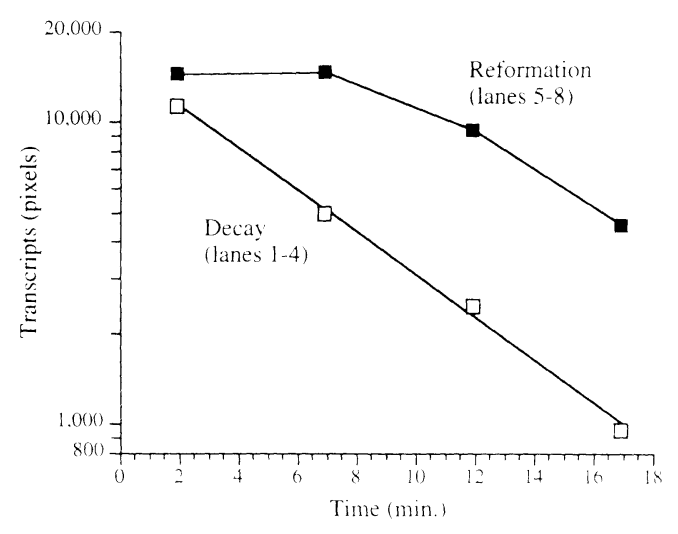

Figure 3. Decay of purified open complexes and their reformation. $(A)$ Open complexes were formed at $37^{\circ} \mathrm{C}$ on a linear template $(1-\mathrm{kb}$ PvuII fragment; see Materials and methods) carrying a variant of the $g \ln A$ promoter region with four A/T to G/C transitions at positions $-2,-3,-5$, and -6 (pJES760; transcript length $\sim 154$ bases; see Materials and methods for details). The reaction was terminated by addition of heparin, and then open complexes were purified away from free proteins and ATP by sieving at $4^{\circ} \mathrm{C}$. The fraction containing the majority of the template was split into three portions; ATP was added back to each, NTRC and NTRB were added back to the second and third portions to the same levels used to form open complexes initially, and a 17-fold excess of a second linear template yielding a shorter transcript (" - 10 to -2 heteroduplex promoter"; transcript length 112 bases) was added to the third portion. The three reaction mixtures were then shifted back to $37^{\circ} \mathrm{C}$, and after 2 min samples were taken at 5 -min intervals and added to elongation mix to allow any polymerase molecules in open complexes to synthesize single transcripts. An autoradiograph is shown of the polyacrylamide gel on which transcripts were isolated. (Lanes 1-4) The decay of open complexes from the first template at 2, 7, 12, and $17 \mathrm{~min}$, respectively. (Lanes 5-8) The reformation of open complexes on the first template at the same times. (Lanes 9-12) The reformation of open complexes on the second template as they decay from the first. $(B)$ The data shown in lanes $1-8$ of $A$ were quantitated as described in Materials and methods and plotted on a logarithmic scale as a function of time. ( $\square$ ) Transcripts in lanes $1-4$ (decay of open complexes from the first template); ( transcripts in lanes 5-8 (reformation of open complexes on the first template). $(C)$ The data shown in lanes $9-12$ of $A$ were quantitated and plotted as in $B$. $(O)$ Transcripts from the first template (decay of open complexes from the first template in the presence of the second); $(\mathbf{O})$ transcripts from the second template (reformation of open complexes on the second template); $(\Delta)$ the sum of the transcripts from both templates. Pixel values for transcripts on the second template have been multiplied by 1.4 to correct for the fact that these templates contain fewer $C$ residues 150 rather than 70 ; see Materials and methods). The conversion factor from pixels to fmoles is $1.6 \times 10^{-5}$.

ture of a heteroduplex. As detailed below, transcriptionally competent complexes formed in the absence of NTRC differed from those formed in its presence with respect to choice of initiating nucleotide, stability, and the requirement for conserved promoter sequences.
Open complexes at the $\operatorname{gln} A$ promoter initiated transcription at both the $A$ residue at +1 and the $G$ residue at +3 (Fig. 4B, lane 1; Popham et al. 1991). Open complexes formed on the heteroduplex promoters in the presence of NTRC also initiated at both of these residues 
A

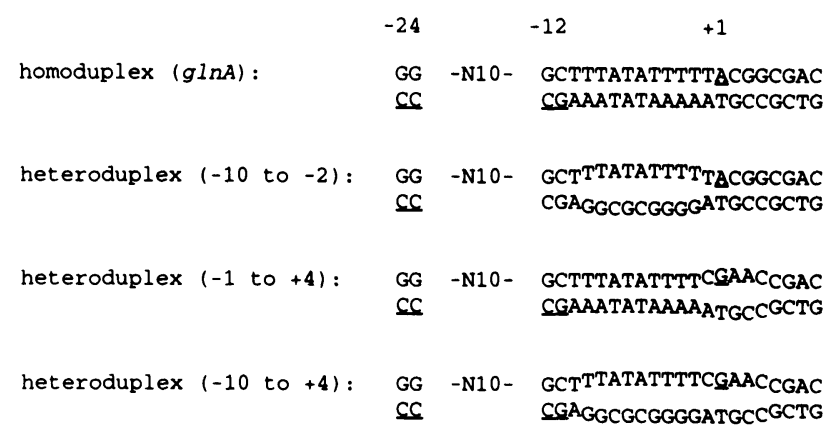

B

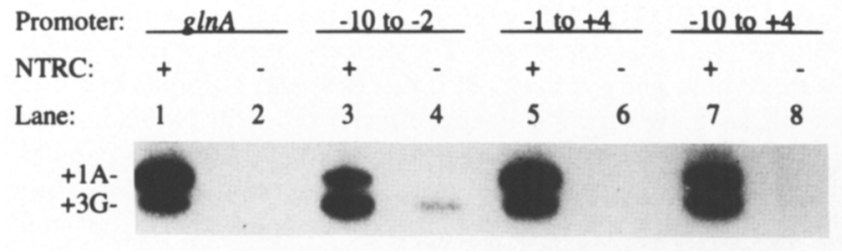

Figure 4. Requirement for NTRC for transcript synthesis from preopened templates. $(A)$ Templates. Each template (700-bpPvuII-BstXI fragment; see Materials and methods) carried a promoter-regulatory region containing the $\operatorname{gn} A$ enhancer at a distance of $450 \mathrm{bp}$ upstream of the transcription start site. The template labeled homoduplex carries the wild-type $g \ln A$ promoter-regulatory region, whereas the templates labeled heteroduplex contain regions of heteroduplex between the promoter and the transcriptional start site $(-10$ to -2$)$ or between the promoter and a position just downstream of the transcriptional start site $(-1$ to +4 and -10 to +4$)$. Heteroduplexes were constructed by purifying individual single strands from phagemid particles, followed by renaturation and digestion with a restriction enzyme that releases the promoter-bearing fragment (see Materials and methods). Because the heteroduplexes were reconstituted from purified single strands, they are free of the corresponding alternative heteroduplexes. In all cases, transcripts lack $U$ residues, which minimizes background transcription by core polymerase originating at the fragment ends. $|B|$ Transcription. Transcription was carried out using the above templates $(1 \mathrm{nM})$ and $50 \mathrm{nM} \sigma^{54}$-holoenzyme (50 nM core polymerase and $\left.200 \mathrm{nM}^{54}\right)$. Reaction mixtures yielding transcripts shown in the odd lanes also contained wild-type NTRC and NTRB (100 and $20 \mathrm{nM}$, respectively). Templates are indicated above the lanes. Shown is an autoradiograph of the gel on which transcripts were isolated.

(Fig. 4B, lanes 3, 5, and 7). In contrast, transcriptionally competent complexes formed on the heteroduplex promoters in the absence of NTRC initiated primarily at +3 (see especially Fig. 4B, lane 4), providing a first line of evidence that these complexes are not on the normal reaction pathway.

To confirm that the two sorts of complexes formed between $\sigma^{54}$-holoenzyme and the heteroduplex promoters initiated differently, we labeled the $5^{\prime}$ ends of such transcripts formed at the promoter carrying heterodu- plex between -10 and -2 with $\left[\gamma^{-32} \mathrm{P}\right] \mathrm{ATP}$ or $\left[\gamma^{-32} \mathrm{P}\right] \mathrm{GTP}$ to identify transcripts initiating at +1 or +3 , respectively; we also labeled with $\left[\alpha{ }^{-32} \mathrm{P}\right] \mathrm{CTP}$, as usual, to detect all transcripts. As at the wild-type $g \ln A$ promoter, complexes between $\sigma^{54}$-holoenzyme and the heteroduplex promoter that were formed in the presence of NTRC initiated at both +1 and +3 (Fig. 5A, lanes 1-3). However, complexes formed in the absence of NTRC initiated almost exclusively at +3 (Fig. 5A, lanes 4-6). Because the same high concentration of ATP was present in reaction mixtures with or without NTRC, the failure of complexes formed in the absence of NTRC to initiate at the $+1 \mathrm{~A}$ residue did not result from a lack of substrate. It is particularly interesting that the complexes formed without NTRC preferred to initiate at the +3 rather than +1 position because base mispairing in the template extended only through -2 with respect to the start site of transcription. Nevertheless, $\sigma^{54}$-holoenzyme alone had access to the template a few bases downstream of the end of the heteroduplex region. The same effect of NTRC on the start point of initiation was obtained with the templates carrying heteroduplex between -1 and +4 and between -10 and +4 (not shown).

To compare the stabilities of transcription complexes formed in the presence or absence of NTRC, we formed them on the promoter carrying heteroduplex from -10 to -2 at a subsaturating concentration of $\sigma^{54}$-holoenzyme and in the presence of ATP. We then diluted them 50-fold into buffer containing heparin to minimize the formation of new complexes. After a further 30-min incubation at $37^{\circ} \mathrm{C}$, complexes formed in the presence of NTRC had decayed by only 1.6 -fold relative to the undiluted control (i.e., $36 \%$ of the complexes had decayed; Fig. 5B, cf. lane 2 with lane 1), whereas those formed in the absence of NTRC had decayed by 90 -fold to near background (i.e., $99 \%$ of the complexes had decayed; Fig. $5 B$, cf. lane 4 with lane 3). Similar results were obtained with complexes formed on the promoters carrying heteroduplex from -1 to +4 and -10 to +4 (not shown).

To provide further evidence that transcription complexes formed without NTRC on heteroduplex promoters are not on the natural reaction pathway, we constructed a "mutant" version of the -10 to -2 heteroduplex promoter lacking the conserved GG dinucleotide in the -24 region (deleted from -27 to -18 ; see Materials and methods). In the absence of NTRC, transcription from this mutant promoter was the same as that from the parental heteroduplex (Fig. 5C, lanes 2,4). However, whereas NTRC greatly stimulated transcription from the parental promoter (Fig. 4B, cf. lane 3 with lane 4; Fig. 5C, cf. lane 1 with lane 2), it had no effect on transcription from the mutant promoter (Fig. 5C, cf. lane 3 with lane 4). This indicates that initiation of transcription from the parental heteroduplex promoter in the $a b$ sence of NTRC and ATP results from a complex that is not dependent on the conserved promoter recognition sequence in the -24 region and therefore lies outside the normal reaction pathway.

Because the behavior of transcription complexes formed on heteroduplex promoters in the absence of 


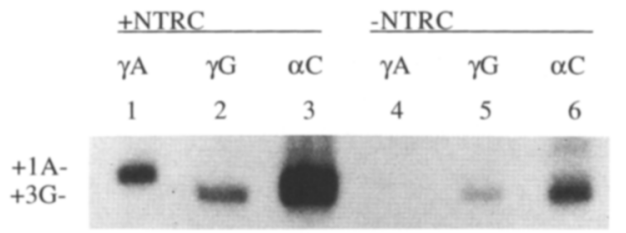

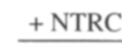

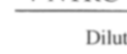

2

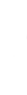

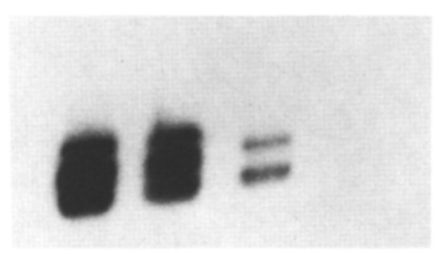

C

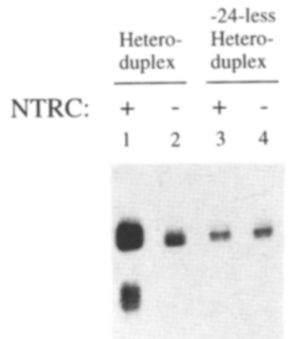

Figure 5. Complexes between $\sigma^{54}$-holoenzyme and the -10 to -2 heteroduplex promoter have different properties depending on whether they are formed in the presence or absence of NTRC: $\langle A|$ Transcriptional start site; $(B)$ stability; $(C)$ requirement for conserved promoter sequences. (A) Start site. The -10 to -2 heteroduplex promoter (700-bp PvuII-BstXI fragment; $3.75 \mathrm{~nm}$; Fig. $4 \mathrm{~A}$ ) and $25 \mathrm{~nm}$ $\sigma^{54}$-holoenzyme $\left(25 \mathrm{~nm}\right.$ core polymerase and $\left.80 \mathrm{~nm} \sigma^{54}\right)$ were incubated for $10 \mathrm{~min}$ at $37^{\circ} \mathrm{C}$ with (lanes $\left.1-3\right)$ or without (lanes 4-6) added wild-type NTRC and NTRB (20 nM each) in a volume of $40 \mu \mathrm{l}$. Then a nucleotide mixture $(10 \mu l)$ containing different labeled nucleotides was added. Final concentrations of nucleotides were $200 \mu \mathrm{M}$, ATP and GTP; $400 \mu \mathrm{M}$, CTP. The labeled nucleotides were $\left|\gamma^{-32} \mathrm{P}\right| \mathrm{ATP}(\sim 60 \mu \mathrm{Ci}$, lanes 1,4$) ;\left(\gamma^{-32} \mathrm{P} \mid \mathrm{GTP}(\sim 60 \mu \mathrm{Ci}\right.$, lanes 2,5$) ;\left|\alpha^{-32} \mathrm{P}\right| \mathrm{CTP}(\sim 9 \mu \mathrm{Ci}$, lanes 3,6$)$. The concentrations of nucleotides provided were sufficient for phosphorylation of NTRC, open complex formation, and synthesis of transcripts. An autoradiograph is shown of the gel on which transcripts were isolated. $(B)$ Stability. Complexes between $\sigma^{54}$-holoenzyme and the -10 to -2 heteroduplex promoter (1-kb PVuII fragment; see Materials and methods) were formed with or without NTRC ${ }^{\mathrm{D} 54 \mathrm{E} \text {, S160F }}$ in two parallel reactions, each containing ATP at $400 \mu \mathrm{M}$. Each reaction mixture was then split; one portion was immediately provided with elongation mix containing both ADP and heparin; the other was diluted 50-fold into a buffer lacking polyethylene glycol and containing ADP at $100 \mu \mathrm{M}$ and heparin at $100 \mu \mathrm{g} / \mathrm{ml}$ and incubated for a further $30 \mathrm{~min}$ at $37^{\circ} \mathrm{C}$ before elongation mix was added. The initial formation of complexes between $\sigma^{54}$-holoenzyme and the promoter was carried out with limiting core RNA polymerase (0.5 nM) so that the forward association reaction of holoenzyme with the promoter would be minimized after dilution (to $0.01 \mathrm{~nm}$ ), allowing any difference in the stabilities of complexes formed with or without NTRC to be detected. Elongation was carried out with the same nucleotide concentrations and the same specific activity of the labeled nucleotide in each case, and transcripts were precipitated from the entire reaction mixture and run on a denaturing gel; hence, all lanes are directly comparable. The presence or absence of NTRC and the use of dilution are indicated above the lanes. Shown are autoradiographs of the gels on which transcripts were isolated. There are four transcript bands rather than the two seen in $A$ because the template was not digested with BstXI and hence there are two stops within the bank of $\mathrm{T}$ residues used for termination, as well as two starts. $|C|$ Requirement for conserved promoter sequences. Complexes were formed between $60 \mathrm{nM} \sigma^{54}$-holoenzyme $\left(60 \mathrm{nM}\right.$ core polymerase and $\left.180 \mathrm{nM} \sigma^{54}\right)$ and template (1-kb PvuII fragments; $0.8 \mathrm{nM}$ ) with or without NTRC ${ }^{\mathrm{S} 160 \mathrm{~F}}$ and NTRB (each at $20 \mathrm{nM}$ ). Formation of complexes and their assessment by transcription were as described in $B$ and Materials and methods, except that ADP was not added with heparin before elongation. Because heparin does not disrupt complexes between $\sigma^{54}$-holoenzyme and heteroduplex promoters, the prominent short transcript band in lane 1 is probably attributable to a second cycle of initiation. Templates were the -10 to -2 heteroduplex promoter $(1$ anes 1,2$)$ or the -10 to -2 heteroduplex promoter lacking conserved promoter sequences in the -24 region (lanes 3,4; see Materials and methods). The templates were not digested with BstXI (see legend to B). NTRC and NTRB were present for the samples in lanes 1 and 3, as indicated.

NTRC is unlike that of natural open complexes with respect to choice of the transcriptional start site, stability, and requirements for conserved promoter sequences, preopening the template around the transcriptional start site by base mispairing does not appear to substitute for the function of NTRC. Therefore, the rate-limiting step in open complex formation cannot lie solely in melting of the DNA.

Interestingly, transcription from heteroduplex promoters in the absence of NTRC was dependent on $\sigma^{54}$; core polymerase alone did not yield detectable transcripts (not shown). Moreover, $\sigma^{70}$-holoenzyme initiated transcription very efficiently from the heteroduplex promoters, although they carry no recognizable -35 or -10 region. The latter result is congruent with the finding that $\sigma^{70}$-holoenzyme can transcribe from a preopened $\lambda$ $p_{\mathrm{RM}}$ promoter in the direction opposite of normal, as well as in the normal direction (Tripatara and deHaseth 1993). Taken together, the results indicate that holoen- zyme forms of RNA polymerase can transcribe from preopened templates without going through the normal open complex intermediate (see also Aiyar et al. 1994).

\section{Discussion \\ There is a high kinetic barrier to open complex formation by $\sigma^{54}$-holoenzyme}

$\sigma^{54}$-Holoenzyme differs from $\sigma^{70}$-holoenzyme in having a high kinetic (activation energy) barrier to open complex formation. Even in the presence of all four nucleotides, $\sigma^{54}$-holoenzyme is essentially incapable of transcript synthesis in vitro in the absence of the activator NTRC (Fig. 2A, lanes 6,7; Popham et al. 1989). This indicates that $\sigma^{54}$-holoenzyme alone cannot form open complexes even transiently. In contrast, as far as we are aware, $\sigma^{70}$-holoenzyme can synthesize transcripts in vitro in the absence of auxiliary factors at all promoters 
that have been tested; these include promoters that are activator dependent in vivo and at which open complex formation is activator dependent under stringent conditions in vitro (e.g., the lac promoter; R. Ebright, per. comm.l, promoters at which open complexes do not accumulate sufficiently to be detected physically (e.g., the trp promoter; Kierkegaard et al. 1983), and promoters at which physical detection of open complexes depends on the presence of initiating nucleotides (e.g., the $\operatorname{rrnB} \mathrm{Pl}$ promoter; Newlands et al. 1991; Ohlsen and Gralla 1992).

There is a thermodynamic barrier to open complex formation by $\sigma^{54}$-holoenzyme

In addition to the kinetic barrier to open complex formation by $\sigma^{54}$-holoenzyme, there is a thermodynamic barrier. That is, in the absence of ATP hydrolysis by NTRC, the position of equilibrium favors closed complexes. As indicated above, open complexes do not form in the absence of NTRC or ATP hydrolysis. Moreover, we have now demonstrated that open complexes formed in the presence of both of these factors and then separated from them decay back to closed complexes, as opposed to inactive polymerase (Fig. 3). Because NTRC does not remain associated with the polymerase in open complexes, a thermodynamic requirement indicates that there must be a mechanism for coupling ATP hydrolysis by NTRC to open complex formation by $\sigma^{54}$-holoenzyme. In this sense NTRC acts as a simple molecular machine (Alberts and Miake-Lye 1992). In contrast, nucleotide hydrolysis by G proteins plays a regulatory role (Bourne et al. 1991; Webb 1992). Binding of nucleoside triphosphate confers an "active conformation" on G proteins, whereas binding of nucleoside diphosphate yields an inactive conformation. In the case of NTRC, neither ATP $\gamma$ S nor $\mathrm{ADP}$ yields an active conformation; rather, nucleotide hydrolysis is required for activity (Weiss et al. 1991).

\section{NTRC is not a site-specific helicase}

We have considered two possible stages for the involvement of NTRC in open complex formation by $\sigma^{54}$-holoenzyme. One is that NTRC acts as a site-specific helicase to perform the DNA strand-denaturation reaction per se, a mode of action that has been assumed by others (Laurent et al. 1993). A second possibility is that NTRC couples the energy of nucleotide hydrolysis to a change in conformation of $\sigma^{54}$-holoenzyme and that this conformational change enables the polymerase itself to carry out the strand-denaturation reaction. We have obtained evidence against the first possibility by showing that preopened promoter templates do not circumvent the requirement for either NTRC or ATP, although they are used very effectively if both are provided (Figs. 4 and 5). Hence, DNA strand separation does not appear to be the rate-limiting step in open complex formation by $\sigma^{54}$-holoenzyme and NTRC is not a site-specific helicase. Therefore, we take as a working hypothesis that NTRC mediates a conformational change in polymerase. Such a conformational change is known to accompany (i.e., to occur before or concomitant with) open complex formation, as evidenced by a lengthening of the polymerase "footprint" to a position downstream of the transcriptional start site (Fig. 1; Popham et al. 1989).

The inferred mode of action of NTRC is of interest with respect to the mechanism of open complex formation by $\sigma^{70}$-holoenzyme and $\sigma^{32}$-holoenzyme. Kinetic studies indicate that a conformational change in these polymerases is rate limiting for open complex formation, rather than denaturation of the DNA strands per se $(\mathrm{Ka}-$ desch et al. 1982; Buc and McClure 1985; Roe et al. 1985; Duval-Valentin and Ehrlich 1987; for review, see Buc 1989; Leirmo and Record 1989). This conformational change characterizes the transition from the "first closed complex," an initial weak-binding recognition complex, to the "second closed complex," a tighter binding intermediate complex that is formed before DNA melting. The second closed complex has been characterized physically at temperatures too low to allow DNA strand separation $\left[10-20^{\circ} \mathrm{C}\right.$ for linear templates; Spassky et al. (1985) for lacUV5; Hofer et al. (1985) for fd PVIII; DuvalValentin and Ehrlich (1986) for pSC101 tet ${ }^{\mathrm{R}}$; Li and McClure (1988) for $\lambda p_{\mathrm{RM}}$ (up-1, $\Delta 265$ ); Schickor et al. (1990) for T7/A1; Cowing et al. (1989) and Mecsas et al. (1991) for $g r o E]$. In it, polymerase usually has a longer footprint than in the initial closed complex, and this footprint extends downstream of the transcriptional start site; lengthening of the footprint is inferred to precede DNA strand denaturation at higher temperatures.

\section{The ATP requirement differs from that for initiation of transcription by eukaryotic RNA polymerase II}

In contrast to the case for $\sigma^{54}$-holoenzyme, the requirement for ATP hydrolysis for initiation of transcription by eukaryotic RNA polymerase II is circumvented by use of a supercoiled plasmid template or a preopened linear template (Parvin and Sharp 1993; Tantin and Carey 1994; Timmers 1994). Use of a supercoiled plasmid template also obviates the need for the general transcription factor TFIIH, which has an ATP-dependent helicase activity (Schaeffer et al. 1993). (Requirements for general transcription factors have not yet been determined with preopened linear templates (Tantin and Carey 1994).] At present it is not clear whether the helicase activity of TFIIH is required for open complex formation per se, promoter clearance, or both (Wang et al. 1992; Parvin and Sharp 1993; Goodrich and Tiian 1994; Timmers 1994).

\section{Materials and methods}

Templates

Plasmid pJES750 is derived from pJES463, which carries the $\operatorname{gln} A$ promoter-regulatory region containing a number of synthetic restriction sites and yields a transcript containing $64 \mathrm{C}$ residues (Porter et al. 1993; Wedel 1993). In pJES750, the sequence between the Bcll site at position -96 of PJES463 and the Ncol site at -38 (containing the low-affinity NTRC binding 
sites III, IV, and V) has been replaced with the 403-bp BclI-NcoI fragment from pACYC184, positioning NTRC-binding sites I and II, which constitute the $g \ln A$ enhancer, at -484 and -452 with respect to the start site of transcription (Porter et al. 1993). In addition, pJES750 contains four tandem oligonucleotides lacking $\mathrm{T}$ residues in the nontranscribed strand (ACCACA. GACCACACCACAGAC) that were inserted into the BstXI site of pJES463 at +28 (the site was regenerated). When UTP is omitted during the elongation phase of a single-cycle transcription assay, transcription from pJES750 terminates at about position +113 , the beginning of a bank of $T$ residues in the nontranscribed strand, yielding a transcript of 112 bases containing $50 \mathrm{C}$ residues. If pJES750 is cleaved with BstXI, the runoff transcript contains 108 bases and $49 \mathrm{C}$ residues.

Plasmids pJES752, pJES754, and pJES755 are the same as pJES750 with the exception of the bases in the melting region downstream of the promoter. In pJES752, the sequence ATTTT from positions -6 to -2 is replaced with GCTCC; in PIES754, the sequence TTATATTTT from positions -10 to -2 is replaced with CCGCGCCCC; and in pJES755, the sequence TACGG from -1 to +4 is replaced with CGAAC. The base changes were made by PCR using the NcoI site at -38 and the HindIII site in the polylinker downstream of the transcript terminus. pJES760 is the same as pJES752, except that it carries six rather than four tandem oligonucleotides and hence yields a transcript of $\sim 154$ bases containing $70 \mathrm{C}$ residues.

Plasmids pJES761 and pIES762 are the same as pJES750 and pJES754, respectively, with the exception of bases in the -24 region of the promoter. In both cases, PCR was used to delete bases -27 through -18 .

\section{Heteroduplex templates}

Plasmid pJES750 was constructed in the vector pTZ19U/Mead et al. 1986), which produces and packages the template strand when replicated from the phage $\mathrm{fl}$ origin. The EcoRI-HindIII fragments bearing the promoter regions from pJES750, pJES754, pJES755, and pJES763 were also cloned into pTZ19R, which produces the opposite strand when replicated from the $\mathrm{fl}$ origin. Single-stranded plasmid carrying each of the three promoter regions was made from the constructs in both pTZ19U and pTZ19R (Ausubel et al. 1989) and purified using Qiagen tip-500 columns (Qiagen, cat. no. 12162). Two complementary single strands (10 $\mu \mathrm{g}$ each) were heated to $95^{\circ} \mathrm{C}$ for $10 \mathrm{~min}$ in $800 \mu \mathrm{l}$ of full-strength PvuII buffer (New England Biolabs buffer 2) and slowly cooled to room temperature. Then 100 units of $P v u I I$ was added, and digestion was allowed to proceed for $2 \mathrm{hr}$ at $37^{\circ} \mathrm{C}$. DNA was precipitated with ethanol, and the 1 -kb $P_{v u I I}$ frag. ment bearing the heteroduplex promoter region was purified on an agarose gel. After isolation from the gel, templates used for the experiments of Figures 3, 4, and 5A were digested with Bst XI, which cleaves at the $3^{\prime}$ end of the transcript-coding sequence just before the bank of $T$ residues. When $A, C$, and $G$ nucleotides are provided, the resulting 700-bp fragment yields a runoff transcript with a single $3^{\prime}$ end. It was important that the concentration of single-stranded DNA in the annealing step be low to avoid formation of a large network of partially annealed DNA circles.

\section{Proteins}

Wild-type NTRC from Salmonella typhimurium, the NTRC $^{\text {constitutive }}$ proteins NTRC ${ }^{\text {S160F }}$ and NTRC D54E, S160F, NTRB, and $\sigma^{54}$ were purified as described (Keener and Kustu 1988; Popham et al. 1991; Weiss et al. 1991; Klose et al. 1993).
Core RNA polymerase from Escherichia coli was kindly provided by D. Hager and R. Burgess (University of Wisconsin, Madison).

\section{Open complex formation}

Formation of open complexes was detected in a single-cycle transcription assay, as detailed below. Studies from several laboratories have established the validity of this assay by showing that the requirements for the formation of open complexes are the same whether this assay is used to detect them or any one of four additional assays is used (e.g., Sasse-Dwight and Gralla 1988; Popham 1989; Popham et al. 1989; Weiss et al. 1991; Wedel 19931.

Core RNA polymerase and $\sigma^{54}$ were mixed together on ice, to which buffer [final concentration, $50 \mathrm{~mm}$ HEPES-acetate $(\mathrm{pH}$ 8.0 ), $100 \mathrm{~mm}$ potassium acetate, $8 \mathrm{~mm}$ magnesium acetate, 27 $\mathrm{mm}$ ammonium acetate, $1 \mathrm{~mm}$ dithiothreitol, and $3.5 \%$ (wt/vol) polyethylene glycol (6000-8000; Sigma), final pH 8.0), template and, if present, NTRC and NTRB, were added. The final concentrations of template and protein components for individual experiments are detailed in the figure legends.

Mixtures were then warmed to $37^{\circ} \mathrm{C}$ for $10 \mathrm{~min}$, and reactions were initiated by adding ATP to a final concentration of $0.4 \mathrm{~mm}$ in a total volume of $24 \mu \mathrm{l}$. After $10 \mathrm{~min}$, formation of open complexes was terminated by addition of heparin to a final concentration of $0.1 \mathrm{mg} / \mathrm{ml}$ and ADP to a final concentration of 4 $\mathrm{mm}$. After an additional $10 \mathrm{~min}$, nucleotides were added to allow synthesis of transcripts [GTP to $0.4 \mathrm{~mm}$ and CTP to $0.1 \mathrm{~mm}$; $5 \mathrm{mCi}$ of $\left[\alpha^{-32} \mathrm{P} \mid \mathrm{CTP}\right.$ was also added $(10 \mathrm{mCi} / \mathrm{mmole}$, New England Nuclear|]. After $10 \mathrm{~min}$, transcripts were precipitated and isolated by gel electrophoresis. Gels were dried and transcript bands were quantified using a Molecular Dynamics PhosphorImager. Any deviations from this protocol are detailed in the relevant figure legend.

The specific activity of CTP was determined by spotting $1 \mu \mathrm{l}$ of a $1: 1000$ dilution of the heparin-nucleotide mix on the dried gel before visualization/quantitation on the PhosphorImager. The specific activity of transcripts was then determined by multiplying the specific activity of CTP by the number of $\mathrm{C}$ residues per transcript-70 for the transcript from pJES760 and 50 for transcripts from pJES750, pJES752, pIES754, pJES755, and each of the templates carrying a region of heteroduplex downstream of the promoter ("heteroduplex promoter templates"). Finally, radioactivity in transcript bands (pixel volume corrected for background) was converted to fmoles of transcript.

\section{Decay and reformation of open complexes}

Open complexes were formed on a Pvull fragment of $\sim 1 \mathrm{~kb}$ derived from pIES760, as detailed below; this template yields a transcript of $\sim 154$ bases containing $70 \mathrm{C}$ residues. Core RNA polymerase (50 nM), $\sigma^{54}(200 \mathrm{~nm})$, NTRC (100 nM), NTRB (20 $\mathrm{nM})$, and template (10 $\mathrm{nm}$ ) were mixed in a final volume of $140 \mu \mathrm{l}$ in the transcription buffer described above and incubated at $37^{\circ} \mathrm{C}$ for $10 \mathrm{~min}$. Formation of open complexes was then initiated by addition of ATP to $1 \mathrm{~mm}$. After a further incubation of $8 \mathrm{~min}$, heparin was added to $100 \mu \mathrm{g} / \mathrm{ml}$ and the mixture was chilled on ice. To assess the number of open complexes at this point, a $20-\mu l$ sample was taken and incubated with elongation mix to allow synthesis of transcripts.

To separate open complexes from free proteins, $100 \mu \mathrm{l}$ of the mixture described above was loaded on a $24-\mathrm{ml}$ Superose- 12 column (Pharmacia HR 10/30 prepacked) that had been equilibrated in transcription buffer without polyethylene glycol, and open complexes were eluted at $0.3 \mathrm{ml} / \mathrm{min}$ in the same buffer. The column fraction containing the bulk of the template and, 
hence, of open complexes, was identified by absorbance at 260 $\mathrm{nm}$ and contained DNA at a concentration of $0.3 \mathrm{nM}$. To monitor decay and reformation of open complexes, the column fraction identified above was split into three portions of $80 \mu \mathrm{l}$ each on ice. Each portion was made $1 \mathrm{~mm}$ in ATP and $3.5 \%$ in polyethylene glycol, the second and third portions were also made $100 \mathrm{~nm}$ in NTRC and $20 \mathrm{nM}$ in NTRB, and the third portion was made $5 \mathrm{nM}$ /representing an excess of 17 -fold over the first template) in the -10 to +4 heteroduplex promoter template, which yields a transcript of $\sim 113$ bases containing $50 \mathrm{C}$ residues (see Fig. 3). The heteroduplex template was used because $\sigma^{54}$-holoenzyme binds to it with a very low apparent $K_{\mathrm{D}}$ (Wedel 1993). The final volume of all three portions was $100 \mu$ l. The three mixtures were transferred to prewarmed glass tubes at $37^{\circ} \mathrm{C}$ to initiate the decay and reformation reactions. Beginning $2 \mathrm{~min}$ after transfer to $37^{\circ} \mathrm{C}, 20-\mu \mathrm{l}$ samples were removed at 5 -min intervals and added to an elongation mix containing heparin, GTP, and CTP plus $\alpha^{32}$ P|CTP to allow any open complexes to form transcripts. This procedure takes a "snapshot" of the number of open complexes at any given moment, and in portion 3 , it shows the partitioning of holoenzyme between the two templates.

Before sieving on the Superose- 12 column, $30 \%$ of the template molecules carried polymerase in an open complex, whereas after sieving that fraction was $\sim 3 \%$, meaning that $\sim 10 \%$ of the open complexes survived the $45 \mathrm{~min}$ between addition of heparin before loading the column and collection of the template fraction off the column. The half-life of open complexes on this template is $\sim 30 \mathrm{~min}$ at $0^{\circ} \mathrm{C}$ in the absence of heparin and is shorter in the presence of heparin. Addition of heparin was found to be necessary to prevent free holoenzyme from remaining associated with the template during passage through the column, and the open complexes were therefore in contact with heparin until some time after they entered the column.

Control experiments indicated that incubation of polymerase with template at low concentrations of each $(0.01 \mathrm{nM}$ core polymerase, $0.01 \mathrm{nM} \sigma^{54}$ and $0.3 \mathrm{nM}$ template in the presence of polyethylene glycol; conditions of Fig. 3A, lanes 5-8) resulted in no loss of polymerase activity after $1 \mathrm{hr}$ at $37^{\circ} \mathrm{C}$. Hence, loss of activity in lanes 5-8 of Figure 3A cannot be accounted for on this basis.

\section{Acknowledgments}

We thank Anne North for expert help with preparation of the figures. This work was supported by National Institutes of Health grant GM38361 to S.K.

The publication costs of this article were defrayed in part by payment of page charges. This article must therefore be hereby marked "advertisement" in accordance with 18 USC section 1734 solely to indicate this fact.

\section{References}

Aiyar, S.E., J.D. Helmann, and P.L. deHaseth. 1994. A mismatch bubble in double-stranded DNA suffices to direct precise transcription initiation by Escherichia coli RNA polymerase. J. Biol. Chem. 269: 13179-13184.

Alberts, B. and R. Miake-Lye. 1992. Unscrambling the puzzle of biological machines: The importance of the details. Cell 68: 415-420.

Ausubel, F.M., R. Brent, R.E. Kingston, D.D. Moore, J.G. Seidman, J.A. Smith, and K. Struhl. 1989. In Current protocols in molecular biology, vol. 1, Greene Publishing Associates/ Wiley-Interscience, New York.

Bourne, H.R., D.A. Sanders and F. McCormick. 1991. The GTP-ase superfamily: Conserved structure and molecular mechanism. Nature 349: 117-127.

Buc, H. 1989. Initiation of prokaryotic transcription-kinetic and structural approaches. In Nucleic acids and molecular biology (ed. F. Eckstein and D.M.J. Lilley), pp. 186-195. Springer-Verlag, Berlin, Germany.

Buc, H. and W.R. McClure. 1985. Kinetics of open complex formation between Escherichia coli RNA polymerase and the lac UV5 promoter. Evidence for a sequential mechanism involving three steps. Biochemistry 24: 2712-2723.

Buck, M. and W. Cannon. 1992. Specific binding of the transcription factor sigma-54 to promoter DNA. Nature 358: $422-424$

Cannon, W., F. Claverie-Martin, S. Austin, and M. Buck. 1994. Identification of a DNA-contacting surface in the transcription factor sigma-54. Mol. Microbiol. 11: 227-36.

Cowing, D.W., J. Mecsas, M.T.J. Record, and C.A. Gross. 1989 Intermediates in the formation of the open complex by RNA polymerase holoenzyme containing the sigma factor sigma ${ }^{32}$ at the groE promoter. J. Mol. Biol. 210: 521-530.

Dixon, R., T. Eydmann, N. Henderson, and S. Austin. 1991. Substitutions at a single amino acid residue in the nitrogenregulated activator protein NTRC differentially influence its activity in response to phosphorylation. Mol. Microbiol. 5: 1657-1667.

Duval-Valentin, G. and R. Ehrlich. 1986. Interaction between $E$. coli RNA polymerase and the tetR promoter from pSC101: Homologies and differences with other $E$. coli promoter systems from close contact point studies. Nucleic Acids Res. 14: 1967-1983.

. 1987. Dynamic and structural characterisation of multiple steps during complex formation between $E$. coli RNA polymerase and the tetR promoter from pSC101. Nucleic Acids Res. 15: 575-594.

Flashner, Y., D.S. Weiss, J. Keener, and S. Kustu. 1995. Constitutive forms of the enhancer-binding protein NtrC: Evidence that essential oligomerization determinants lie in the central activation domain. J. Mol. Biol. 249: 700-713.

Goodrich, J.A. and R. Tjian. 1994. Transcription factors IIE and IIH and ATP hydrolysis direct promoter clearance by RNA polymerase II. Cell 77: 145-156.

Hellman, J.D. and M.J. Chamberlin. 1988. Structure and function of bacterial sigma factors. Annu. Rev. Biochem. 57: 839-872.

Hofer, B., D. Muller, and H. Koster. 1985. The pathway of E. coli RNA polymerase-promoter complex formation as visualized by footprinting. Nucleic Acids Res. 13: 5995-6013.

Kadesch, T.R., S. Rosenberg, and M.J. Chamberlin. 1982. Binding of Escherichia coli RNA polymerase holoenzyme to bacteriophage T7 DNA. I. Mol. Biol. 155: 1-29.

Keener, J. and S. Kustu. 1988. Protein kinase and phosphoprotein phosphatase activities of nitrogen regulatory proteins NTRB and NTRC of enteric bacteria: Roles of the conserved amino-terminal domain of NTRC. Proc. Natl. Acad. Sci. 85: 4976-4980.

Kierkegaard, K., H. Buc, A. Spassky, and J.C. Wang. 1983. Mapping of single-stranded regions in duplex DNA at the sequence level: Single-strand-specific cytosine methylation in RNA polymerase-promoter complexes. Proc. Natl. Acad. Sci. 80: 2544-2548.

Klose, K.E., D.S. Weiss, and S. Kustu. 1993. Glutamate at the site of phosphorylation of nitrogen-regulatory protein NTRC mimics aspartyl-phosphate and activates the protein. J. Mol. 
Biol. 232: 67-78.

Kustu, S., E. Santero, J. Keener, D. Popham, and D. Weiss. 1989. Expression of sigma 54 (ntrA)-dependent genes is probably united by a common mechanism. Microbiol. Rev. 53: 367376.

Kustu, S., A.K. North, and D.S. Weiss. 1991. Prokaryotic transcriptional enhancers and enhancer-binding proteins. Trends Biochem. Sci. 16: 397-402.

Laurent, B.C., I. Treich, and M. Carlson. 1993. The yeast SNF2/ SW12 protein has DNA-stimulated ATPase activity required for transcriptional activation. Genes \& Dev. 7: 583-591.

Leirmo, S. and M.T. Record Jr. 1989. Structural, thermodynamic and kinetic studies of the interaction of $\mathrm{E}^{70} \mathrm{RNA}$ polymerase with Promoter DNA. In Nucleic acids and molecular biology (ed. F. Eckstein and D.M.J. Lilley), pp. 123-151. Springer-Verlag, Berlin, Germany.

Li, X. and W.R. McClure. 1988. Closed complexes at the $\lambda \mathrm{P}_{\mathrm{RM}}$ promoter. In DNA-protein interactions in transcription led. J. Gralla), pp. 63-72. Alan R. Liss, New York.

Lonetto, M., M. Gribskov, and C.A. Gross. 1992. The sigma 70 family: Sequence conservation and evolutionary relationships. J. Bacteriol. 174: 3843-3849.

Magasanik, B. 1993. The regulation of nitrogen utilization in enteric bacteria. I. Cell. Biochem. 51: 34-40.

Mead, D.A., E. Szczesna-Skorupa, and B. Kemper. 1986. Singlestranded DNA "blue" T7 promoter plasmids: A versatile tandem promoter system for cloning and protein engineering. Prot. Eng. 1: 67-74.

Mecsas, J., D.W. Cowing, and C.A. Gross. 1991. Development of RNA polymerase-promoter contacts during open complex formation. J. Mol. Biol. 220: 585-597.

Merrick, M.J. 1993. In a class of its own-the RNA polymerase sigma factor $\sigma^{54}\left(\sigma^{\mathrm{N}}\right)$. Mol. Microbiol. 10: 903-909.

Newlands, J.T., W. Ross, K.K. Gosink, and R.L. Gourse. 1991. Factor-independent activation of Escherichia coli rRNA transcription. II. Characterization of complexes of $\operatorname{rrnB~Pl}$ promoters containing or lacking the upstream activator region with Escherichia coli RNA polymerase. I. Mol. Biol. 220: $569-583$.

Ohlsen, K.L. and J.D. Gralla. 1992. DNA melting within stable closed complexes at the Escherichia coli rrnB P1 promoter. I. Biol. Chem. 267: 19813-19818.

Parvin, J.D. and P.A. Sharp. 1993. DNA topology and a minimal set of basal factors for transcription by RNA polymerase II. Cell 73: 533-540.

Popham, D. L. 1989. "Biochemical studies on the activation of nitrogen-regulated transcription in enteric bacteria." $\mathrm{Ph}$. D. thesis, University of California, Davis, CA.

Popham, D.L., D. Szeto, J. Keener, and S. Kustu. 1989. Function of a bacterial activator protein that binds to transcriptional enhancers. Science 243: 629-635.

Popham, D., J. Keener, and S. Kustu. 1991. Purification of the alternative $\sigma$ factor, $\sigma^{54}$, from Salmonella typhimurium and characterization of $\sigma^{54}$-holoenzyme. I. Biol. Chem. 266: 19510-19518.

Porter, S.C., A.K. North, A.B. Wedel, and S. Kustu. 1993. Oligomerization of NTRC at the $g \ln A$ enhancer is required for transcriptional activation. Genes \& Dev. 7: 2258-2273.

Porter, S.C., A.K. North, and S. Kustu. 1995. Mechanism of transcriptional activation by NTRC (Nitrogen Regulatory Protein C): Requirement for oligomerization and phosphorylation. In Two-component signal transduction (ed. T. Silhavy and J. Hoch), pp. 147-158. American Society for Microbiology, Washington, D.C.

Roe, J.H., R.R. Burgess, and M.T. Record. 1985. Temperature dependence of the rate constants of the Escherichia coli RNA polymerase-lambda $P_{R}$ promoter interaction. Assignment of the kinetic steps corresponding to protein conformational change and DNA opening. I. Mol. Biol. 184: 441453.

Sanders, D.A., C.B. Gillece, A.L. Burlingame, and D.J. Koshland. 1992. Phosphorylation site of NtrC, a protein phosphatase whose covalent intermediate activates transcription. I. Bacteriol. 174: 5117-5122.

Sasse-Dwight, S. and J.D. Gralla. 1988. Probing the Escherichia coli glnALG upstream activation mechanism in vivo. Proc. Nat. Acad. Sci. 85: 8934-8938.

Schaeffer, L., R. Roy, S. Humbert, B. Moncollin, W. Vermeulen, J.H. Hoeijmakers, P. Chambon, and J.M. Egly. 1993. DNA repair helicase: A component of BTF2 (TFIIH) basic transcription factor. Science 260: 58-63.

Schickor, P., W. Metzger, W. Werel, H. Lederer, and H. Heumann. 1990. Topography of intermediates in transcription initiation of E. coli. EMBO I. 9: 2215-2220.

Spassky, A., K. Kirkegaard, and H. Buc. 1985. Changes in the DNA structure of the lac UV5 promoter during formation of an open complex with Escherichia coli RNA polymerase. Biochemistry 24: 2723-2731.

Tantin, D. and M. Carey. 1994. A heteroduplex template circumvents the energetic requirement for ATP during activated transcription by RNA polymerase II. J. Biol. Chem. 269: $17397-17400$.

Thony, B. and H. Hennecke. 1989. The $-24 /-12$ promoter comes of age. FEMS Microbiol. Rev. 63: 341-358.

Timmers, H.T. 1994. Transcription initiation by RNA polymerase II does not require hydrolysis of the beta-gamma phosphoanhydride bond of ATP. EMBO J. 13: 391-399.

Tripatara, A. and P.L. deHaseth. 1993. A new start site for Escherichia coli RNA polymerase at an engineered short region of non-complementarity in double-stranded DNA. I. Mol. Biol. 233: 349-358.

Wang, W., M. Carey, and J.D. Gralla. 1992. Polymerase II promoter activation: Closed complex formation and ATPdriven start site opening. Science 255: 450-453.

Webb, M.R. 1992. The role of nucleoside triphosphate hydrolysis in transducing systems: p21ras and muscle. Philos. Trans. R. Soc. Lond. Biol. Sci. 336: 19-24.

Wedel, A.B. 1993. "The mechanism of open complex formation by $\sigma^{54}$-holoenzyme." Ph. D. thesis. University of California, Berkeley, CA.

Weglenski, P., A.J. Ninfa, S. Ueno-Nishio, and B. Magasanik. 1989. Mutations in the $\ln G$ gene of Escherichia coli that result in increased activity of nitrogen regulator I. I. Bacteriol. 171: 4479-4485.

Weiss, D.S., J. Batut, K.E. Klose, J. Keener, and S. Kustu. 1991. The phosphorylated form of the enhancer-binding protein NTRC has an ATPase activity that is essential for activation of transcription. Cell 67: 155-167.

Weiss, V. and B. Magasanik. 1988. Phosphorylation of nitrogen regulator I (NRI) of Escherichia coli. Proc. Natl. Acad. Sci. 85: 8919-8923.

Whitehall, S., S. Austin, and R. Dixon. 1992. DNA supercoiling response of the sigma 54-dependent Klebsiella pneumoniae nifL promoter in vitro. J. Mol. Biol. 225: 591-607. 


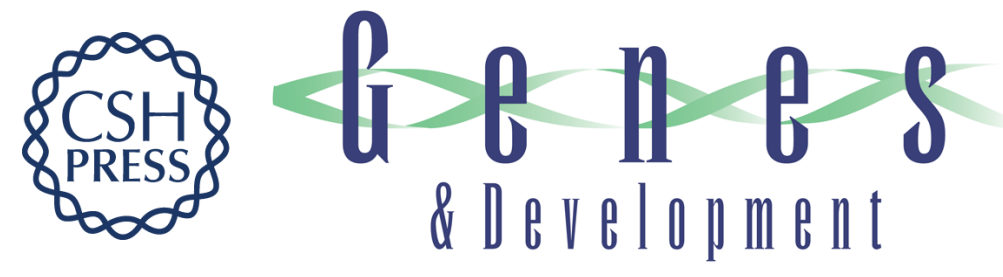

\section{The bacterial enhancer-binding protein NTRC is a molecular machine: ATP hydrolysis is coupled to transcriptional activation.}

A Wedel and S Kustu

Genes Dev. 1995, 9:

Access the most recent version at doi:10.1101/gad.9.16.2042

References This article cites 48 articles, 17 of which can be accessed free at: http://genesdev.cshlp.org/content/9/16/2042.full.html\#ref-list-1

License

Email Alerting Receive free email alerts when new articles cite this article - sign up in the box at the top Service right corner of the article or click here.

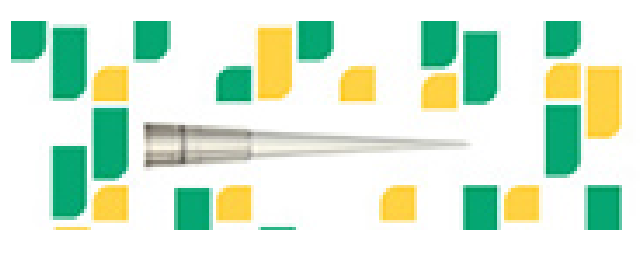

Focused on your science. 